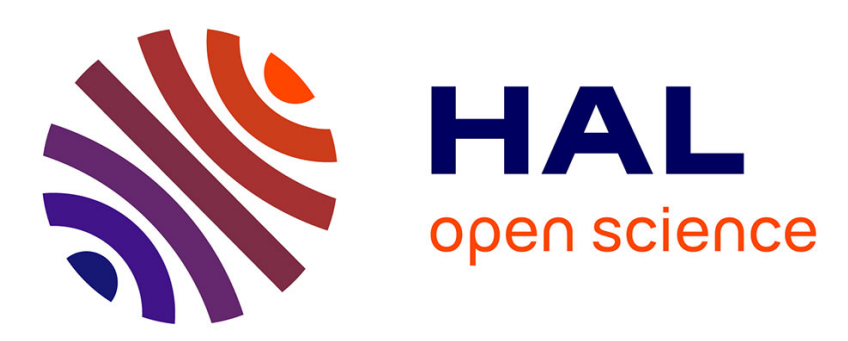

\title{
Influence de l'addition de sels de calcium et du chauffage sur les diverses formes de calcium dans le lait
}

J. Zuraw, Z. Smietana, J. Szpendowski, W. Chojnowski

\section{To cite this version:}

J. Zuraw, Z. Smietana, J. Szpendowski, W. Chojnowski. Influence de l'addition de sels de calcium et du chauffage sur les diverses formes de calcium dans le lait. Le Lait, 1986, 66 (4), pp.421-429. hal-00929079

\section{HAL Id: hal-00929079 https://hal.science/hal-00929079}

Submitted on 1 Jan 1986

HAL is a multi-disciplinary open access archive for the deposit and dissemination of scientific research documents, whether they are published or not. The documents may come from teaching and research institutions in France or abroad, or from public or private research centers.
L'archive ouverte pluridisciplinaire HAL, est destinée au dépôt et à la diffusion de documents scientifiques de niveau recherche, publiés ou non, émanant des établissements d'enseignement et de recherche français ou étrangers, des laboratoires publics ou privés. 


\title{
Influence de l'addition de sels de calcium et du chauffage sur les diverses formes de calcium dans le lait
}

\author{
J. ZURAW, Z. SMIETANA, J. SZPENDOWSKI et W. CHOJNOWSKI \\ Institut du Génie et de la Biotechnologie alimentaires \\ Université Agrotechnique d'Olsztyn, Pologne
}

\section{Résumé}

L'addition au lait de 1,8 à $5,4 \mathrm{mM} /$ litre de chlorure de calcium avant chauffage à 75,85 et $92^{\circ} \mathrm{C}$ pendant $15 \mathrm{~s}$ et $15 \mathrm{~min}$ provoque des changements dans les proportions de calcium (soluble, collö̈dal et ionisé), en comparaison avec celles des échantillons de lait chauffés sans addition de sels de calcium.

Dans le lait enrichi de $3,6 \mathrm{mM}$ de $\mathrm{CaCl}_{2}$, par litre pasteurisé à $92^{\circ} \mathrm{C} / 15 \mathrm{~s}$, les pourcentages des diverses formes de calcium sont proches de celles du lait cru ou non, additionné de sel de calcium, bien que $25 \%$ environ du calcium ionique aient été déplacés au cours de chauffage.

On a constaté que les ions calcium, ajoutés au lait avant une pasteurisation haute et rapide, assurent le maintien de l'aptitude à la coagulation par la présure à un niveau comparable à celle d'un lait ayant subi une pasteurisation ménagée qui est d'usage en fabrication fromagère.

Mots clés : Lait - Calcium - Chauffage.

\section{Summary}

Influence of the addition of calcium salts and of heating on the various forms of calcium in milk

By adding 1.8 to $5.4 \mathrm{mM} / \mathrm{dm}^{3}$ of calcium salts to milk before warming it at 75 , 85 and $92^{\circ} \mathrm{C}$ for $15 \mathrm{~s}$ and $900 \mathrm{~s}$ changes, were caused in amounts of various forms of calcium (soluble, colloidal and ionic), as compared with milk samples to which calcium salts were not added before warming.

In milk which was enriched by $3,6 \mathrm{mM}$ of $\mathrm{CaCl}_{2}$, and pasteurized at $92^{\circ} \mathrm{C}$ for $15 \mathrm{~s}$ the percentage of various forms of calcium approximated their amounts in raw or mildly pasteurized milk, to which no salts of calcium was added, though about $25 \%$ of ionic calcium was displaced in the soluble or colloidal form in the course of warming.

The ions of calcium, as added to milk before HTST pasteurization caused the rennetability of milk to be maintained at the same level as in slightly pasteurized milk, as generally used in cheese manufacture.

Key words : Milk - Calcium - Heat-treatment. 


\section{Introduction}

Le calcium est présent dans le lait, partie à l'état colloïdal, partie en solution, sous forme de sels et d'ions libres (PyNe, 1962; BloOMfield et Moor, 1973 ; PIERRE et Brulé, 1981). L'équilibre entre ces différentes formes peut être modifié par le chauffage, un changement de température ou d'acidité ainsi que par addition de certains sels inorganiques (ZitTle, 1969 ; Melachouris, 1972 ; Brulé et FAUQUANT, 1981). L'état d'équilibre des formes calciques influe sur les caractères physico-chimiques du lait, les dimensions des micelles de caséine, la stabilité et d'une façon plus générale sur le comportement technologique du lait (MoRRISSEY, 1969 ; KNOOP et al., 1973 ; Moor, 1975 ; SMIETANA et al., 1977 ; SMIETANA, 1979). C'est surtout l'aptitude à la coagulation par la présure qui dépend des teneurs en ions libres et en phosphate de calcium colloïdal.

Ainsi Muldon et LisKa (1972) ont observé qu'il existe une relation entre la fermeté du coagulum et la teneur du lait en calcium ionisé dont la teneur ne doit pas être inférieure à $1,5 \mathrm{mM} /$ litre.

Dans nos travaux précédents, nous avons constaté qu'en ajoutant au lait une petite quantité d'ions calcium avant la pasteurisation à température ambiante pendant un temps court, on pouvait obtenir une bonne fermeté du gel et une récupération plus complète des protéines de lait (PozNANSKI et al., 1972 ; SMIETANA, 1979).

Dans la présente étude nous avons essayé de déterminer les influences conjuguées de l'addition des sels de calcium et du chauffage sur les équilibres entre les diverses formes de calcium.

\section{Matériel et méthodes}

Le lait cru écrémé de la ferme universitaire, divisé en portions, était additionné d'une solution de chlorure de calcium pur, en quantités variant de 1,8 à $5,4 \mathrm{mM}$ de $\mathrm{CalCl}_{2}$ par litre de lait. Des sels de calcium ont été ajoutés juste avant la pasteurisation.

Les échantillons préparés étaient chauffés aux températures de 75,85 et $92^{\circ} \mathrm{C}$ pendant $15 \mathrm{~s}$ et $15 \mathrm{~min}$.

Le traitement des échantillons est présenté dans le schéma suivant:

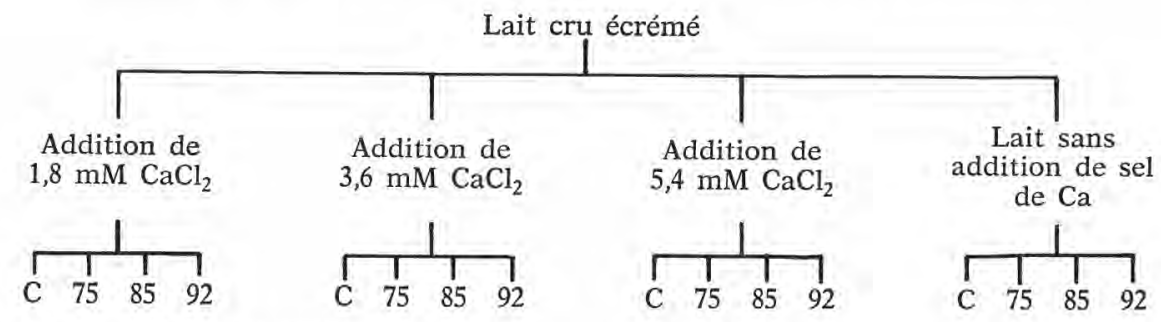

$\mathrm{C}=$ échantillons de lait cru.

$75,85,92=$ échantillons de lait pasteurisé à 75,85 et $92^{\circ} \mathrm{C}$ pendant $15 \mathrm{~s}$ et $15 \mathrm{~min}$. 
Sur les échantillons de lait cru et de lait chauffé, refroidis à $20^{\circ} \mathrm{C}$, les formes suivantes de calcium étaient déterminées : (1954) ;

- calcium total, d'après la méthode décrite par Mattson et Swartling

- calcium «colloïdal», quantité de calcium présente dans le sédiment obtenu par centrifugation d'un volume de lait de $34,5 \mathrm{ml}$ à $68000 \mathrm{~g}$, pendant $35 \mathrm{~min}$, à $37^{\circ} \mathrm{C}$;

- calcium «soluble», quantité de calcium présente dans le surnageant de l'ultracentrifugation précédente ; (1973).

— calcium « ionisé », déterminé à l'aide d'un ionoanalyse ORION-407A

L'aptitude du lait à la coagulation par la présure a été déterminée par la méthode décrite par EISELE et al. (1962).

La fermeté du coagulum était appréciée par détermination des forces tangentielles agissant sur une sphère se déplaçant d'un mouvement uniforme dans l'échantillon; elle est exprimée en unités arbitraires (gramme d'eau).

\section{Résultats et discussion}

Les échantillons de lait cru analysés contenaient en moyenne $31,8 \mathrm{mM}$ de calcium par litre, dont $21,2 \mathrm{mM}$ à l'état colloïdal, $10,5 \mathrm{mM}$ sous forme soluble et $1,68 \mathrm{mM}$ à l'état ionisé.

Bloomfield et Moor (1973), en mettant en œuvre une autre technique de séparation et de détermination de sels de calcium, ont relevé des valeurs proches pour les diverses formes de calcium du lait. Demott (1969), utilisant également une électrode spécifique, a constaté la présence de 1,4 à $2,5 \mathrm{mM}$ de calcium ionique par litre de lait cru écrémé.

Les quantités de sels de calcium ajoutées $\left(1,8\right.$ à $5,4 \mathrm{mM}$ de $\left.\mathrm{CaCl}_{2}\right)$ font augmenter la teneur en calcium total proportionnellement à la quantité de calcium introduite, tout en modifiant en même temps l'équilibre entre les diverses formes. Le taux de calcium soluble augmente de la quantité équivalente de calcium ajouté, tandis que la teneur en calcium colloïdal ne subit pas de changements appréciables; elle reste au même niveau que dans le lait cru non additionné de sel de calcium.

L'addition de sel de calcium fait également augmenter la teneur en calcium ionisé mais cette augmentation n'est pas proportionnelle à la quantité introduite. On constate aussi que le taux de calcium ionisé par rapport au calcium soluble ne change pas d'une manière significative en fonction de la quantité de sel de calcium ajoutée (tabl. 1).

Le chauffage des échantillons de lait non additionnés de sels de calcium à $75-92^{\circ} \mathrm{C}$ pendant $15 \mathrm{~s}$ et $15 \mathrm{~min}$ provoque une diminution des teneurs en calcium soluble et ionisé, par comparaison avec les échantillons de lait cru (tabl. 1). Ainsi avec le traitement $92^{\circ} \mathrm{C}-15 \mathrm{~min}$ il se produit un déplacement de $1,5 \mathrm{mM}$ de cal- 
Tableau I

Concentration des diverses formes de calcium dans le lait

Repartition of calcium salts in milk in function of heating and of previous $\mathrm{CaCl}_{2}$ addition

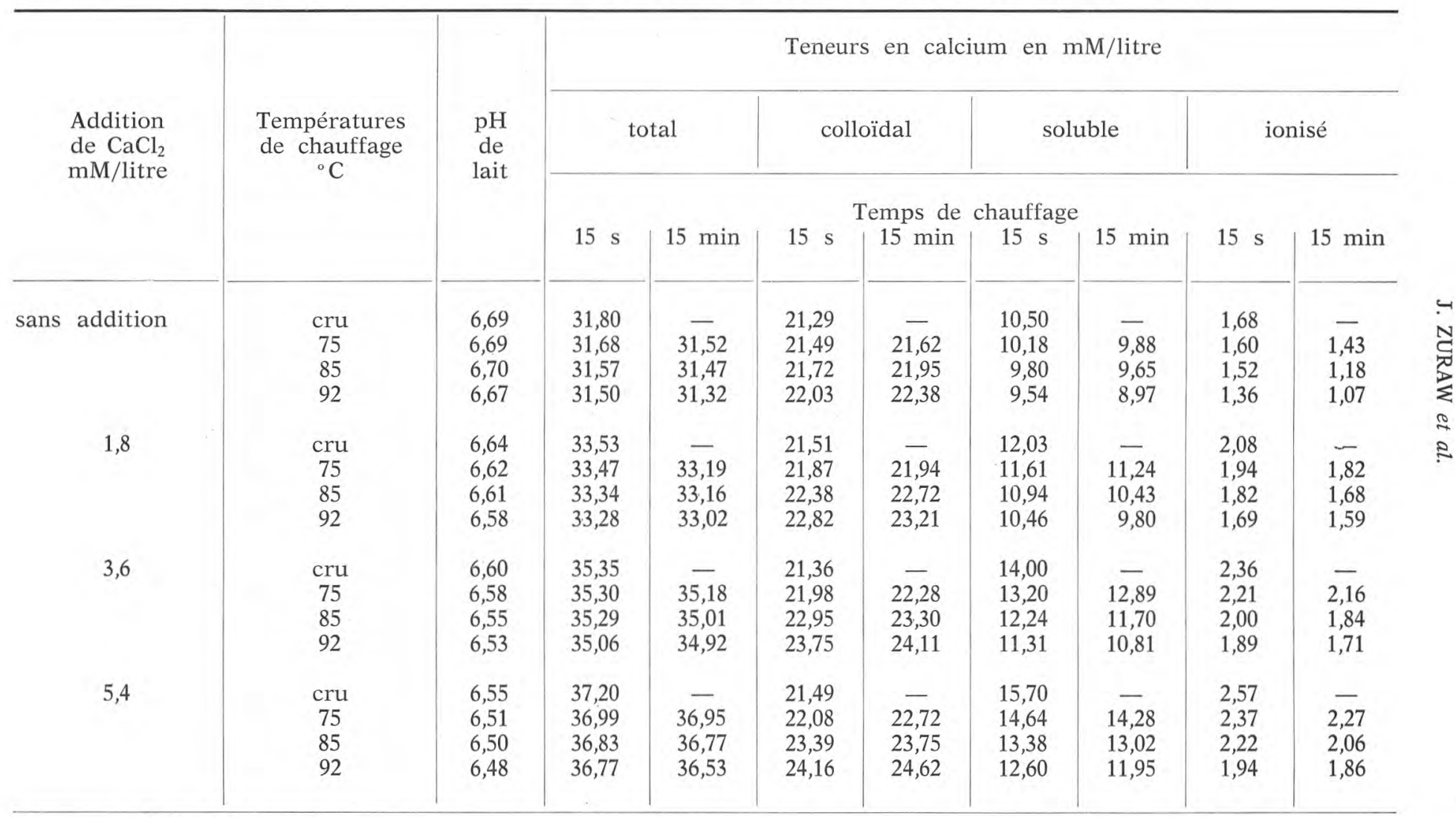

Moyennes de 5 expériences. 


\section{TABLEAU II}

Variations des concentrations des formes de calcium

dans le lait pasteurisé après addition de sels de calcium par rapport à celles dans le lait cru

Variations of calcium salts repartition in function of heating and of previous $\mathrm{CaCl}_{2}$ addition

\begin{tabular}{|c|c|c|c|c|c|c|c|c|c|}
\hline \multirow{3}{*}{$\begin{array}{l}\text { Addition } \\
\text { de } \mathrm{CaCl}_{2} \\
\mathrm{mM} / \text { litre }\end{array}$} & \multirow{3}{*}{$\begin{array}{l}\text { Températures } \\
\text { de chauffage } \\
{ }^{\circ} \mathrm{C}\end{array}$} & \multicolumn{8}{|c|}{ Variations des teneurs en calcium en $\mathrm{mM}$} \\
\hline & & \multicolumn{2}{|c|}{ total } & \multicolumn{2}{|c|}{ colloïdal } & \multicolumn{2}{|c|}{ soluble } & \multicolumn{2}{|c|}{ ionisé } \\
\hline & & \multicolumn{8}{|c|}{ Temps de chauffage } \\
\hline * sans addition & $\begin{array}{l}\text { cru } \\
75 \\
85 \\
92\end{array}$ & $\begin{array}{r}31,80 \\
-0,12 \\
-0,23 \\
-0,30\end{array}$ & $\begin{array}{r}-\overline{0}, 28 \\
-0,33 \\
-0,48\end{array}$ & $\begin{array}{r}21,29 \\
0,20 \\
0,43 \\
0,72\end{array}$ & $\begin{array}{l}-\overline{3} \\
0,66 \\
1,09\end{array}$ & $\begin{array}{r}10,50 \\
-0,32 \\
-0,70 \\
-0,96\end{array}$ & $\begin{array}{c}-\overline{0}, 62 \\
-0,85 \\
-1,53\end{array}$ & $\begin{array}{r}1,68 \\
-0,08 \\
-0,16 \\
-0,32\end{array}$ & $\begin{array}{r}-\overline{0}, 25 \\
-0,50 \\
-0,61\end{array}$ \\
\hline 1,8 & $\begin{array}{l}\text { cru } \\
75 \\
85 \\
92\end{array}$ & $\begin{array}{l}1,73 \\
1,67 \\
1,54 \\
1,48\end{array}$ & $\begin{array}{l}- \\
1,39 \\
1,36 \\
1,22\end{array}$ & $\begin{array}{l}0,32 \\
0,58 \\
1,09 \\
1,53\end{array}$ & $\begin{array}{l}- \\
0,65 \\
1,43 \\
1,92\end{array}$ & $\begin{array}{r}1,53 \\
1,11 \\
0,44 \\
-0,04\end{array}$ & $\begin{array}{r}\overline{0}, 74 \\
0,07 \\
-0,70\end{array}$ & $\begin{array}{l}0,40 \\
0,26 \\
0,14 \\
0,01\end{array}$ & $\begin{array}{r}\overline{0}, 14 \\
0,00 \\
-0,09\end{array}$ \\
\hline 3,6 & $\begin{array}{l}\text { cru } \\
75 \\
85 \\
92\end{array}$ & $\begin{array}{l}3,55 \\
3,50 \\
3,49 \\
3,26\end{array}$ & $\begin{array}{l}- \\
3,38 \\
3,21 \\
3,12\end{array}$ & $\begin{array}{l}0,07 \\
0,69 \\
1,66 \\
2,46\end{array}$ & $\begin{array}{l}-\overline{09} \\
2,01 \\
2,82\end{array}$ & $\begin{array}{l}3,50 \\
2,70 \\
1,74 \\
0,81\end{array}$ & $\begin{array}{l}\overline{2,39} \\
1,20 \\
0,31\end{array}$ & $\begin{array}{l}0,68 \\
0,53 \\
0,32 \\
0,21\end{array}$ & $\begin{array}{l}\overline{0}, 48 \\
0,16 \\
0,03\end{array}$ \\
\hline 5,4 & $\begin{array}{l}\text { cru } \\
75 \\
85 \\
92\end{array}$ & $\begin{array}{l}5,40 \\
5,19 \\
5,03 \\
4,97\end{array}$ & $\begin{array}{l}\overline{5}, 15 \\
4,97 \\
4,73\end{array}$ & $\begin{array}{l}0,20 \\
0,79 \\
2,10 \\
2,87\end{array}$ & $\begin{array}{l}-\overline{1,43} \\
2,46 \\
3,33\end{array}$ & $\begin{array}{l}5,20 \\
4,14 \\
2,88 \\
2,10\end{array}$ & $\begin{array}{l}- \\
3,78 \\
2,52 \\
1,45\end{array}$ & $\begin{array}{l}0,89 \\
0,69 \\
0,54 \\
0,26\end{array}$ & $\begin{array}{l}0,59 \\
0,38 \\
0,18\end{array}$ \\
\hline
\end{tabular}

* Lait cru sans addition de sels de calcium. 
cium soluble, dont $0,6 \mathrm{mM}$ de calcium ionisé toutefois. Le calcium colloïdal n'augmente pas dans les mêmes proportions, car on observe que la pasteurisation des échantillons, enrichis ou non en sels de calcium, réduit le niveau de calcium total, surtout quand elle comporte un chauffage à $92^{\circ} \mathrm{C}$ pendant $15 \mathrm{~s}$ et $15 \mathrm{mn}$ (tabl. 2). Il est probable qu'une part du calcium originel passe sous forme insoluble et se dépose sur la surface de chauffe de l'échangeur.

Le chauffage des laits additionnés de 1,8 à $5,4 \mathrm{mM}$ de sels de calcium fait passer les formes soluble et ionisée à l'état colloïdal d'une manière plus intense que pour les échantillons ne subissant pas l'addition de calcium.

Avec l'augmentation de la température, de la durée de chauffage et de la quantité de sel de calcium ajouté on observe que le taux de calcium colloïdal s'accroît et qu'il atteint les valeurs maximales respectives de $24,16 \mathrm{mM}$ et $24,62 \mathrm{mM} /$ litre pour les échantillons additionnés de $5,4 \mathrm{mM}$ de sels de calcium, chauffés à $92^{\circ} \mathrm{C}$ pendant $15 \mathrm{~s}$ et $15 \mathrm{~min}$. Il est à noter que dans le lait enrichi de $3,6 \mathrm{mM}$ de $\mathrm{CaCl}_{2}$, chauffé à $92^{\circ} \mathrm{C} / 15 \mathrm{~s}$, les pourcentages des formes diverses de calcium sont proches de celles du lait cru, non additionné de sel de calcium (tabl. 3).

L'augmentation importante du calcium colloïdal est l'indice d'une liaison du calcium avec le complexe caséinate ou avec les protéines du sérum dénaturées. Les teneurs en calcium ionisé indiquent que les doses de calcium ajoutées au lait peuvent assurer après le chauffage un niveau de cette forme de calcium égal ou supérieur à celle présente à l'origine dans le lait cru, bien que le déplacement de calcium ionique au cours du chauffage puisse atteindre $25 \%$ (tabl. 2, 3). Avec des additions de calcium de 1,8 et $3,6 \mathrm{mM} /$ litre et après un chauffage à $92^{\circ} \mathrm{C} / 15 \mathrm{~s}$, on observe que les teneurs en calcium ionisé sont respectivement de 1,69 et $1,89 \mathrm{mM} /$ litre, soit des niveaux proches de celui du lait cru non additionné de sel de calcium $(1,68 \mathrm{mM} /$ litre $)$.

L'appréciation de l'aptitude du lait à la coagulation par la présure témoigne que, malgré la pasteurisation à $92^{\circ} \mathrm{C}$, un lait additionné de chlorure de calcium peut avoir un comportement comparable à celui d'un lait pasteurisé à $75^{\circ} \mathrm{C}$ pensant $15 \mathrm{~s}$ (tabl. 4). Ainsi, sans addition de calcium, le lait qui a subi la pasteurisation la plus sévère donne un gel dont la fermeté $\left(5,2 \mathrm{~g} \mathrm{H}_{2} \mathrm{O}\right)$ a une valeur près de trois fois plus faible que celle du gel de lait cru $\left(13,8 \mathrm{~g} \mathrm{H}_{2} \mathrm{O}\right)$.

En revanche des échantillons de lait additionnés de 1,8 à $5,4 \mathrm{mM}$ de $\mathrm{CaCl}_{2}$, pasteurisés à $92^{\circ} \mathrm{C} / 15 \mathrm{~s}$, coagulent d'une manière semblable au lait pasteurisé à $75^{\circ} \mathrm{C} / 15 \mathrm{~s}$, et les gels formés ont une fermeté de 10,6 à $11,8 \mathrm{~g} \mathrm{H}_{2} \mathrm{O}$ après 42 et $27 \mathrm{~min}$.

MULDON et LisKa (1972) ont rapporté qu'après le chauffage du lait l'aptitude à la coagulation originelle ne pouvait être restaurée par augmentation de la teneur en calcium, même par une addition de $2 \mathrm{mM}$ de $\mathrm{CalCl}_{2} /$ litre.

En revanche les résultats de nos expériences montrent que l'apport d'ions calcium avant une pasteurisation très sévère permet de maintenir le comportement à l'emprésurage au niveau de celui d'un lait ayant subi une pasteurisation ménagée qui est habituelle en fromagerie.

Il semble d'ailleurs que le taux de calcium n'est pas seul à intervenir, mais que tout le système complexe des combinaisons calciques solubles et colloïdales, conservé après le chauffage à un niveau proche de celui du lait cru, conditionne l'aptitude à la coagulation par la présure. PYNE (1962) avait déjà observé qu'une 
TABLEAU III

Variations des proportions des diverses formes de calcium dans le lait pasteurisé

Variations of calcium salts proportions in function of heating and of previous $\mathrm{CaCl}_{2}$ addition

\begin{tabular}{|c|c|c|c|c|c|c|c|c|c|c|c|}
\hline \multirow{2}{*}{$\begin{array}{l}\text { Addition } \\
\text { de } \mathrm{CaCl}_{2} \\
\mathrm{mM} / \text { litre }\end{array}$} & \multirow{2}{*}{$\begin{array}{l}\text { Températures } \\
\text { de chauffage } \\
{ }^{\circ} \mathrm{C}\end{array}$} & \multicolumn{2}{|c|}{ colloïdal } & \multicolumn{2}{|c|}{ soluble } & \multicolumn{2}{|c|}{$\begin{array}{c}\begin{array}{c}\text { du calcium } \\
\text { soluble }\end{array} \\
\text { ionisé }\end{array}$} & \multicolumn{2}{|c|}{$\begin{array}{l}\text { Ca-soluble } \\
\text { Ca-colloïdal }\end{array}$} & \multicolumn{2}{|c|}{$\begin{array}{c}\begin{array}{c}\mathrm{Ca}^{* *} \\
\text { duauffé lait }\end{array} \\
\mathrm{Ca}^{* *} \text { du lait cru }\end{array}$} \\
\hline & & $15 \mathrm{~s}$ & $15 \mathrm{~min}$ & $15 \mathrm{~s}$ & $15 \mathrm{~min}$ & $15 \mathrm{~s}$ & $15 \mathrm{~min}$ & $15 \mathrm{~s}$ & $15 \min$ & $15 \mathrm{~s}$ & $15 \min$ \\
\hline 1,8 & $\begin{array}{l}\text { cru } \\
75 \\
85 \\
92\end{array}$ & $\begin{array}{l}64,15 \\
65,34 \\
67,12 \\
68,56\end{array}$ & $\begin{array}{l}\overline{66,10} \\
68,51 \\
70,29\end{array}$ & $\begin{array}{l}35,87 \\
34,68 \\
32,87 \\
31,43\end{array}$ & $\begin{array}{l}\overline{33,86} \\
31,45 \\
29,67\end{array}$ & $\begin{array}{l}17,29 \\
16,70 \\
16,62 \\
15,96\end{array}$ & $\begin{array}{l}\overline{16,37} \\
16,10 \\
16,02\end{array}$ & $\begin{array}{l}0,56 \\
0,53 \\
0,49 \\
0,46\end{array}$ & $\begin{array}{l}-\overline{50} \\
0,46 \\
0,42\end{array}$ & $\begin{array}{l}1,24 \\
1,15 \\
1,08 \\
0,99\end{array}$ & $\begin{array}{l}\overline{1,08} \\
1,00 \\
0,83\end{array}$ \\
\hline 5,4 & $\begin{array}{l}\text { cru } \\
75 \\
85 \\
92\end{array}$ & $\begin{array}{l}57,76 \\
59,69 \\
63,50 \\
65,70\end{array}$ & $\begin{array}{l}6 \overline{1,49} \\
64,59 \\
67,39\end{array}$ & $\begin{array}{l}42,20 \\
39,57 \\
36,32 \\
34,26\end{array}$ & $\begin{array}{l}-\overline{38,64} \\
35,08 \\
32,71\end{array}$ & $\begin{array}{l}16,36 \\
16,18 \\
16,59 \\
15,39\end{array}$ & $\begin{array}{l}\overline{15,89} \\
15,82 \\
15,56\end{array}$ & $\begin{array}{l}0,73 \\
0,66 \\
0,57 \\
0,52\end{array}$ & $\begin{array}{l}\overline{0,63} \\
0,58 \\
0,48\end{array}$ & $\begin{array}{l}1,52 \\
1,41 \\
1,32 \\
1,15\end{array}$ & $\begin{array}{l}\overline{1,35} \\
1,22 \\
1,10\end{array}$ \\
\hline
\end{tabular}




\section{TABLeaU IV}

Evaluation de l'aptitude des laits à la coagulation par la présure Evaluation of rennet coagulation ability of treated milk

\begin{tabular}{|c|c|c|c|c|}
\hline $\begin{array}{l}\text { Addition } \\
\text { de } \mathrm{CaCl}_{2} \\
\mathrm{mM} / \text { litre }\end{array}$ & $\begin{array}{l}\text { Températures } \\
\text { de chauffage } \\
{ }^{\circ} \mathrm{C}\end{array}$ & $\begin{array}{l}\text { Temps } \\
\text { de chauffage } \\
\text { en secondes }\end{array}$ & $\begin{array}{c}\text { Temps } \\
\text { de coagulation } \\
\text { en minutes }\end{array}$ & $\begin{array}{l}\text { Fermeté } \\
\text { du caillé } \\
\mathrm{g} \mathrm{H}_{2} \mathrm{O}\end{array}$ \\
\hline $\begin{array}{c}\text { sans addition } \\
1,8 \\
3,6 \\
5,4\end{array}$ & $\begin{array}{l}\text { cru } \\
\text { cru } \\
\text { cru } \\
\text { cru }\end{array}$ & $\begin{array}{l}- \\
- \\
-\end{array}$ & $\begin{array}{l}32 \\
22 \\
17 \\
12\end{array}$ & $\begin{array}{l}13,8 \\
14,4 \\
16,8 \\
14,0\end{array}$ \\
\hline sans addition & $\begin{array}{l}75 \\
85 \\
92 \\
92\end{array}$ & $\begin{array}{r}15 \\
15 \\
15 \\
900\end{array}$ & $\begin{array}{l}35 \\
41 \\
60 \\
79\end{array}$ & $\begin{array}{r}11,5 \\
9,4 \\
6,2 \\
5,2\end{array}$ \\
\hline 1,8 & $\begin{array}{l}75 \\
85 \\
92 \\
92\end{array}$ & $\begin{array}{r}15 \\
15 \\
15 \\
900\end{array}$ & $\begin{array}{l}26 \\
29 \\
42 \\
79\end{array}$ & $\begin{array}{r}13,5 \\
11,8 \\
10,6 \\
5,5\end{array}$ \\
\hline 3,6 & $\begin{array}{l}75 \\
85 \\
92 \\
92\end{array}$ & $\begin{array}{r}15 \\
15 \\
15 \\
900\end{array}$ & $\begin{array}{l}21 \\
27 \\
28 \\
51\end{array}$ & $\begin{array}{r}14,1 \\
12,9 \\
11,6 \\
7,1\end{array}$ \\
\hline 5,4 & $\begin{array}{l}75 \\
85 \\
92 \\
92\end{array}$ & $\begin{array}{r}15 \\
15 \\
15 \\
900\end{array}$ & $\begin{array}{l}16 \\
22 \\
27 \\
32\end{array}$ & $\begin{array}{r}13,2 \\
12,5 \\
11,8 \\
6,8\end{array}$ \\
\hline
\end{tabular}

Moyennes de 3 expériences.

quantité insuffisante d'ions calcium et de phosphates de calcium solubles peut rendre le lait complètement incoagulable.

De même, Muldon et Liska (1972) et Smietana (1979) ont relevé qu'un faible taux de calcium ionisé dans le lait chauffé diminue la fermeté du gel présure.

D’après MCGAnN et Pyne (1960), une quantité réduite de calcium colloïdal inhibe la phase de coagulation en conséquence des perturbations causées à la structure micellaire.

La fonction probable des petites quantités de calcium ajoutées avant la pasteurisation à $92^{\circ} \mathrm{C} / 15 \mathrm{~s}$ est d'assurer un niveau élevé de calcium ionique et soluble, égal ou supérieur à celui du lait cru, et d'être une source de formation de calcium colloïdal pendant le chauffage.

L'équilibre calcique qui se rapproche le plus de celui du lait cru ou du lait pasteurisé $\left(75^{\circ} \mathrm{C} / 15 \mathrm{~s}\right)$ a été observé sur les échantillons de lait additionné de $3,6 \mathrm{mM}$ de $\mathrm{CaCl}_{2}$ et chauffé à $92^{\circ} \mathrm{C} / 15 \mathrm{~s}$. Le taux de calcium colloïdal était 
alors de $67,74 \%$ et celui de calcium soluble de $32,25 \%$ par rapport au calcium total (tabl. 3).

L'état d'équilibre entre les diverses formes de calcium et surtout le niveau élevé de calcium ionisé, maintenus dans le lait par l'apport de petites quantités de $\mathrm{CaCl}_{2}$ avant pasteurisation, assurent une bonne coagulation du lait par la présure. Il a d'ailleurs été démontré dans nos précédents travaux (SMIETANA et al., 1977 ; Smietana, 1979) que le niveau des ions calcium influence directement la quantité de phosphate colloïdal et les dimensions des micelles de caséine du lait pasteurisé.

Reçu le 21 octobre 1985.

Accepté pour publication le 26 juin 1986.

\section{Références bibliographiques}

Bloomfield V.A., Moor C.V., 1973. Structure of casein micelles physical methods. Neth. Milk Dairy J., 27, 103-120.

Brulé G., Fauquant J., 1981. Mineral balance in skim-milk and milk retentate : effect of physicochemical characteristic of the aqueous phase. $I$.Dairy., 48, 91-97.

DEMOTT B.J., 1969. Calcium ion concentration in milk whey and $\beta$-lactoglobulin as influenced by ionic strength, added calcium rennet concentration, and heat. J. Dairy Sci., 52, 1672-1675.

EIsele M., Babuchowski K., BUdny J., 1962. Proba analizy podpuszczkowego scinania mleka metoda fizyczna. Zesz. nauk. WSR Otsztyn, 13, 2, 237.

KNOOP A.M., KNOOP E., WIECHEN A., 1973. Electron miscroscopical investigation on the structure of the casein micelles. Neth. Milk Dairy J., 27, 121-127.

Mattson S., Swartling P., 1954. Rep. nr 43, Dairy Dep. of Alnarp Institute.

Mc Gann T.C.A., Pyne G.T., 1960. The colloidal phosphate of milk. III. Nature of its association with casein. J. Dairy Res., 27, 403-417.

Melachouris N., 1972. Interaction of $\beta$-lactoglobulin with polyphosphates. J. Agric. Food Chem., 20, 798-802.

Morr C.V., 1975. Chemistry of milk proteins in food processing. J. Dairy Sci., 58, 977-984.

MoRrisSEy P.A., 1969. The heat stability of milk as affected by variations in $\mathrm{pH}$ and milk salts. J. Dairy Res., 36, 343-351.

Muldoon P.J., Liska B.J., 1972. Relationship between ionized calcium and curd tension in reconstituted nonfat dry milk. J. Dairy Sci., 55, 1300-1301.

ORION RESEARCH, 1973. Analytical methods guide (sixth edition).

PoznANSki S., Smietana Z., SuRAzynski A., Krolik T., 1972. Sposob koagulacji bialek mleka. Pat. PRL- 82692.

Pyne G.T., Mc Gann T.C., 1962. The influence of colloidal phosphate of milk on the rennet coagulation. XVI Int. Dairy Cong., B, 611-616.

PyNe G.T., 1962. Some aspects of the physical chemistry of the salts of milk. J. Dairy Res., $29,101-130$.

Pierre A., Brulé G., 1981. Mineral and protein equilibria between the colloidal and soluluble phases of milk at low temperature. J. Dairy Res., 48, 417-428.

SMIETANA Z., 1979. Studia nad ukierunkowana modyfikacja bialek mleka do celow technologicznych. Zesz. naukk. ART Olsztyn. 14, 123.

Smietana Z., Jakubowski J., Poznanski S., Zuraw J., HoSaja M., 1977. The influence of calcium ions and heat on size changes of casein micelles in milk. Milchwissenschaft, 32, 464-467.

ZiTTLE C.A., 1969. Influence of heat on $\alpha$-casein : Effect on $\alpha_{s}$-casein of concentration on calcium chloride and of sodium chloride. $J$. Dairy Sci., 52, 1356-1358. 